\title{
Lung Clearance Index is Increased in Patients with COPD - LCI Measurements in the Daily Routine
}

\section{Sebastian Fähndrich ${ }^{1}$, Philipp M. Lepper ${ }^{1}$, Franziska Trudzinski ${ }^{1}$, Martina Seibert ${ }^{1}$, Stefan Wagenpfeil ${ }^{2}$ and Robert Bals ${ }^{1^{\star}}$}

${ }^{1}$ Department of Internal Medicine, Pulmonology, Allergology, Intensive Care Medicine, Saarland University Hospital, Homburg, Germany

${ }^{2}$ Institute of Medical Biometry, Epidemiology and Medical Informatics, Saarland University Hospital, Homburg, Germany

\begin{abstract}
Objective: The lung clearance index ( $\mathrm{LCl})$, an index of ventilation homogeneity derived from the multiple breath wash out (MBW) of an inert gas, is a lung function test to monitor pulmonary disease. The aim of this study was to investigate whether LCI measurements are suitable for the daily routine in patients with COPD in comparison to young and healthy controls.

Methods: MBW measurements were performed successfully in 23 patients (out of 42) patients with COPD $\left(\mathrm{FEV}_{1} \%, 40.41 \pm 14.65\right)$. As controls we selected 30 healthy and young volunteers. All subjects used the EasyOne ProLab $^{\mathrm{TM}}$ (ndd, Switzerland).

Results: 18 patients were not able to finish measurements successfully because of significant leaks, cough and irregular breathing (GOLD I $n=1$ (5.6\%); GOLD II $n=6$ (33.3\%); GOLD III $n=5$ (27.7\%); GOLD IV $n=6$ (33.3\%). The mean LCl was significantly higher in COPD patients as compared to young and healthy volunteers $(12.55 \pm 3.50$ vs $7.00 \pm 1.02$, $p<0.05)$. Although LCl correlated with FEV $\%$ of predicted $\left(r^{2}=-0.540, p<0.01\right)$ and $R_{\text {tot }}(\%$ pred. $)\left(r^{2}=0.504\right.$, $p<0.01$ ) the method failed to discriminate between GOLD II-IV classes. LCl correlated with the volume of trapped gas in elderly patients with COPD and young and healthy controls [FRC (\% pred.) $\left(r^{2}=0.191, p<0.01\right)$, ITGV $(\%$ pred.) $\left(r^{2}=0.478, p<0.01\right)$, and RV (\% pred.) $\left.\left(r^{2}=0.462, p<0.01\right)\right]$. Moreover, the results did not correlate with the $6-M W T$, a validated clinical outcome parameter.

Conclusions: Although measurement of $\mathrm{LCl}$ was capable to detect inhomogeneous ventilation (airway obstruction and hyperinflation), this method did not discriminate between GOLD II-IV and did not correlate with 6-MWT. More than $43 \%$ of the patients with COPD did not complete the study because of difficulties with the method. Nevertheless, the $\mathrm{LCl}$ might be a tool to gain insight into lung pathophysiology in younger patients e.g. children. Our preliminary study encourages further investigations for the future.
\end{abstract}

Keywords: Lung clearance index; LCI; COPD; Severity classification; Exacerbations; Life quality

Abbreviations:BMI: Body Mass Index; CCI: Charlson Comorbidity Index; COPD: Chronic Obstructive Pulmonary Disease; DLCO: Diffusion Capacity for Carbon Monoxide; FEV ${ }_{1}$ : Forced Expiratory Flow in One Second; FRC: Functional Residual Capacity; FVC: Forced Vital Capacity; GOLD: Global Initiative for Chronic Obstructive Lung Disease; ITGV: Gas Volume in the Thorax; LCI: Lung Clearance Index; MBW: Multiple Breath Wash Out, MEF 75, MEF 50, MEF 25 (maximum expiratory flow at $75 \%, 50 \%, 25 \%$ of FVC); $\mathrm{R}_{\text {tot }}$ : Total Resistance; RV: Residual Volume; SGRQ: St George's Respiratory Questionnaire; TLC: Total Lung Capacity; VC: Vital Capacity; 6-MWT: 6 Minute Walking Test; \% pred: Percent of Predicted.

\section{Introduction}

Chronic obstructive pulmonary disease (COPD) is a progressive lung disease caused by chronic smoke inhalation and characterized by persistent airflow obstruction to the lungs that is not fully reversible [1]. Severity classification has been based on $\mathrm{FEV}_{1}$, which correlates poorly with clinically relevant outcomes such as health-related quality of life, breathlessness, and exercise capacity [2-7].

Therefore, there is the need of new methods for better assessment of disease severity and health status. Our present study aimed to evaluate the feasibility of multiple breath wash out (MBW) for clinical routine. We asked whether LCI correlates with already well proven clinical outcome parameters for COPD SGRQ, number of exacerbations and 6-MWT.

This method has been developed as a noninvasive tool to obtain insight into ventilation homogeneity [8]. The principle of this technique has been described more than 50 years ago and involves the measurement of the exhalation of an inert tracer gas [9]. In case of nitrogen washout, the test is performed by using $100 \%$ oxygen. If helium or SF6 are used as tracer gases, the washout is usually performed by switching to air breathing and measurement of the tracer gas. The lung clearance index (LCI) is calculated from the washout measurements and represents the multiple of the functional residual capacity (FRC) that is necessary to obtain an end tidal concentration of the tracer gas of $1 / 40$ of the initial concentration ( $\mathrm{LCI}=$ cumulative expired volume/ FRC) [10]. Several studies describe the use of LCI measurements to characterize lung disease, most studies were performed in children as the LCI measurement is non-invasive and does not need active respiratory maneuvers. Studies in infants or children with cystic fibrosis (CF) revealed that MBW and LCI measurements are feasible and able to detect lung disease in an early stage [11-15]. LCI measurements were associated with airway infection, inflammation, pulmonary function tests (PFTs), and ventilation inhomogeneity. Studied in children with asthma showed that the LCI is elevated in this disease [16]. Whereas a recent study showed that LCI is increased in patients with COPD [17],

*Corresponding author: Robert Bals, Department of Internal Medicine Pulmonology, Allergology, Intensive Care Medicine, Saarland University Hospital Homburg, Germany, Tel: +49 684116 23601; Fax: +49 684116 23602; E-mail: robert.bals@uks.eu

Received April 29, 2016; Accepted June 13, 2016; Published June 17, 2016

Citation: Fähndrich S, Lepper PM, Trudzinski F, Seibert M, Wagenpfeil S et al. (2016) Lung Clearance Index is Increased in Patients with COPD - LC Measurements in the Daily Routine. J Pulm Respir Med 6: 354. doi:10.4172/2161 $105 \times .1000354$

Copyright: (c) 2016 Fähndrich S, et al. This is an open-access article distributed under the terms of the Creative Commons Attribution License, which permits unrestricted use, distribution, and reproduction in any medium, provided the original author and source are credited. 
no data exist concerning suitability for the daily use in elderly patients with COPD. An abnormal LCI reflects ventilation inhomogeneity due to airway pathology, suggesting that this measurement could be a suitable noninvasive outcome measure in COPD. Until now, there exist no data about the mechanical handling of the method in the clinical routine of elderly patients with COPD and the relationship to the clinical outcome parameters SGRQ, exacerbation rate, and 6-MWT has not been investigated.

The aim of the present study was to investigate whether LCI measurements are feasible in patients with different $\mathrm{FEV}_{1}$ stages of COPD and whether the LCI is correlated with classical PFTs. We hypothesized that LCI would be elevated in COPD and correlate with clinical outcome parameters such as SGRQ, rate of exacerbations, and 6-MWT.

\section{Methods}

\section{Subjects}

In this prospective, cross-sectional, and observational study, patients were recruited from the Department of Internal Medicine $\mathrm{V}$ from January 2013 to January 2014. The diagnosis of COPD in all patients was based according to the GOLD, definition on a postbronchodilator $\mathrm{FEV}_{1} / \mathrm{FVC}<70 \%$ pred., history of $>10$ pack years smoking, and exclusion of other obstructive lung diseases. As healthy controls we included never smokers with normal pulmonary function tests (PFTs). Exclusion criteria for all individuals were: A respiratory infection within 4 weeks and the need of oxygen. This study was approved by the ethics committee of the Ärztekammer des Saarlandes and informed consent was obtained.

\section{Clinical measurements}

Pulmonary function tests (PFTs) by spirometry and body plethysmography were performed following the ATS/ERS guidelines [18]. Individuals performed a minimum of three $\mathrm{FVC}$ and $\mathrm{FEV}_{1}$ maneuvers, maximum expiratory flows at $75 \%, 50 \%, 25 \%$ of FVC (MEF 75 , MEF 50, MEF 25) was recorded. The residual volume (RV), total lung capacity (TLC), and gas volume in the thorax (ITGV) were determined by body plethysmography. The diffusion capacity for carbon monoxide (DLCO) was determined by the single breath technique following the ERS/ATS guidelines [19].

Smoking status (current or former) and smoking history (packyears) were determined. In a subset of patients, we recorded data on COPD related symptoms (St. George's Respiratory Questionnaire), the presence of comorbidities (Charlson comorbidity index, CCI) [20], the exercise capacity by the 6 minute walking test (6-MWT), and the number of exacerbations during the last 12 months. Exacerbations were defined by use of antibiotics, steroids, or both or admission to the hospital related to worsening respiratory symptoms.

\section{Measurement of the LCI by MBW}

MBW measurements were performed by using an EasyOne ProLab ${ }^{\text {tix }}$ (ndd, Switzerland) according to the manufacturer's description with ambient $\mathrm{N}_{2}$ as tracer gas. Patients with the need of continuous oxygen supplementation were excluded because the pre-test dilution of the ambient $\mathrm{N}_{2}$ in the lungs makes the assessment of the start $\mathrm{N}_{2}$ concentration difficult. MBW/LCI measurement was integrated into a clinical workflow. After taking the medical history, measurement of classical PFTs, the individuals were subjected to MBW testing. $\mathrm{N}_{2}$ was determined by indirect method using the molar mass and the $\mathrm{CO}_{2}$ signal. An MBW measurement was declared invalid if significant leaks, cough or irregular breathing occurred.

The functional residual capacity was also measured using the EasyOne ProLab ${ }^{\text {mix }}$ by analyzing the expired $\mathrm{N}_{2}$ trace. Calculation of the LCI was according to standard guidelines of the ERS/ATS [8].

\section{Limitations of the method}

We stated a high drop-out rate with 18 patients who were not able to finish measurements successfully because of significant leaks, cough and irregular breathing (GOLD I $n=1$ (5.6\%); GOLD II $n=6$ (33\%); GOLD III $n=5$ (27.7\%); GOLD IV $n=6$ (33\%). The time for each measurement took more than 20 minutes (dependent on the grade of ventilation inhomogeneity). Repeats of the measurements were very often refused by the patients because the inhalation of dry oxygen gas seemed to be uncomfortable. Moreover, measurements were very often interrupted by cough which lead-as well as leakages at the mouth piece-to invalid measurements. A further limitation was that wash-out measurements were not possible in patients with the need of oxygen.

Only a small number of patients with GOLD IV $(n=6)$ completed the study (50\% of patients with COPD GOLD IV). Except for two subjects, most of our young and healthy controls were fit to complete the measurements without any disturbances $(n=30)$.

\section{Statistical analysis}

Data are displayed as mean \pm standard deviation. Data were analyzed using SPSS (Version 22, IBM, New York, USA). Overall group comparison between GOLD II-IV and healthy controls was performed by ANOVA-test/Kruskal-Wallis-test. In order to test pairwise median group differences, we used the Mann-Whitney-U-test. Pearson linear correlation analysis between LCI and other continuous variables were performed and the results are presented as $\mathrm{r}^{2}$ and resulting $\mathrm{p}$-values. A $P$ value of 0.05 was regarded as significant.

\section{Results}

\section{Study population}

We obtained LCI data of 23 (out of 41) patients with COPD (BMI $24.62 \pm 4.32$ ). We determined smoking exposure. Patients who complete LCI measurements were former smokers and 14 COPD patients were found to be current smokers (pack years of COPD patient's 59.55 \pm 38.43 years). The mean $\mathrm{FEV}_{1} \%$ pred. was $40.41 \pm 14.65$ (female patients $40.33 \% \pm 15.13 \%$, male patients $40.45 \% \pm 14.50 \%)$. Patients with successful measurements were classified according to $\mathrm{FEV}_{1}$-based GOLD stages as II $(n=5)$ moderate, III $(n=12)$ severe, IV $(n=6)$ very severe (Figure 1). Patients reported no exacerbation in the past 4 weeks. In 15 (of 23) patients with COPD, the number of exacerbations was $1.1 \pm 2.6$ last 12 months. The 30 healthy individuals which finished the wash out measurements successfully ( 2 drop-outs because of cough) were more frequently females $(n=19$, males $n=11)$ with a mean age of $44.66 \pm 14.61$ years. All control subjects (BMI $26.50 \pm 6.18 \mathrm{~kg} / \mathrm{m}^{2}$ ) were never-smokers with a normal $\mathrm{FEV}_{1} \%$ pred. $(105.45 \% \pm 15.07 \%)$. Data on the patients and controls are summarized in Table 1.

\section{Especially patients with GOLD IV failed in valid measurements}

The time for each measurement took more than 20 minutes (dependent on the grade of ventilation inhomogeneity). Repeats of the measurements were very often refused by the patients because the inhalation of dry oxygen gas seemed to be uncomfortable. Moreover, measurements were very often interrupted by cough which lead-as well as leakages at the mouth piece - to invalid measurements. A further 


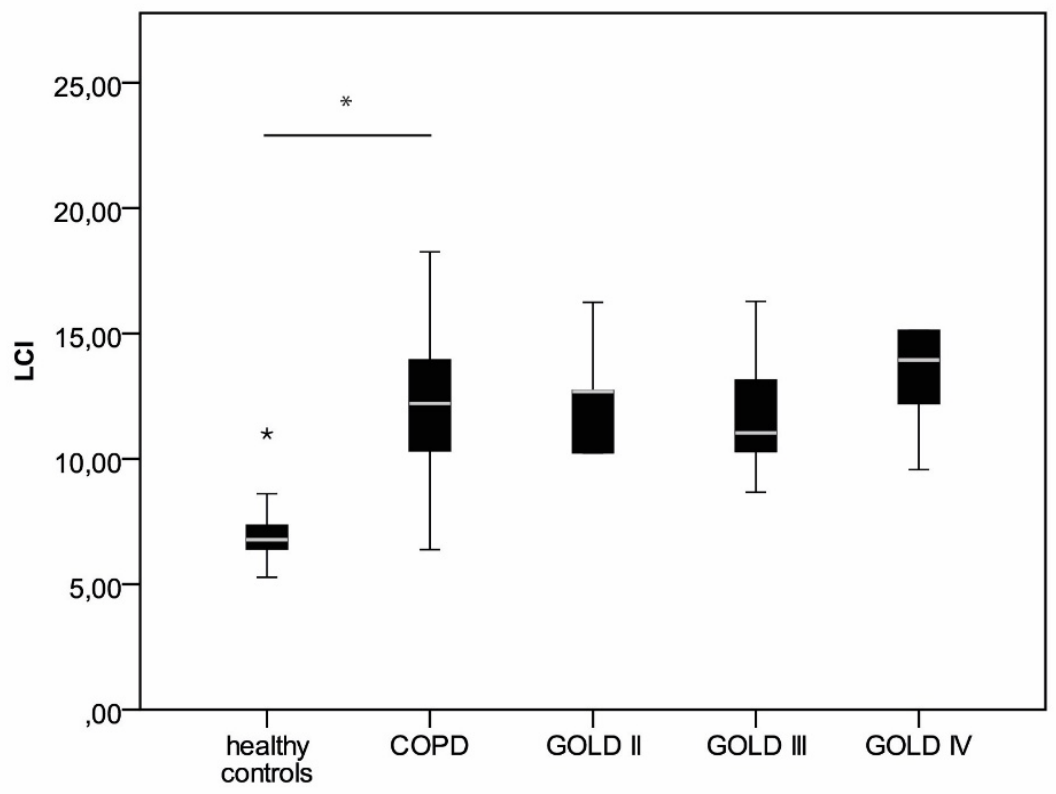

Figure 1: The LCI is increased in patients with COPD (all COPD stages II-IV). There was no difference between the spirometric stages II-IV. ${ }^{*} \mathrm{P}<0.01$.

\begin{tabular}{|c|c|c|c|}
\hline & Patients with COPD & Normal controls & p value \\
\hline age & $62.70 \pm 8.84$ & $44.66 \pm 14.61$ & $<0.05$ \\
\hline height & $169.04 \pm 10.59$ & $171.13 \pm 8.07$ & n.s. \\
\hline weight & $70.86 \pm 15.89$ & $78.11 \pm 21.01$ & n.s. \\
\hline BMI & $24.62 \pm 4.32$ & $26.50 \pm 6.18$ & n.s. \\
\hline packyears & $59.55 \pm 38.43$ & $0.0 \pm 0.0$ & $<0.01$ \\
\hline LCl & $12.55 \pm 3.50$ & $7.00 \pm 1.02$ & $<0.05$ \\
\hline FEV $\%$ & $40.41 \pm 14.65$ & $105.45 \pm 15.07$ & $<0.05$ \\
\hline FRC\% & $58.41 \pm 7.02$ & $47.76 \pm 8.86$ & $<0.01$ \\
\hline VC\% & $79.17 \pm 15.23$ & $105.97 \pm 12.19$ & $<0.05$ \\
\hline ITGV\% & $158.5 \pm 32.94$ & $104.31 \pm 14.53$ & $<0.05$ \\
\hline RV\% & $188.3 \pm 51.19$ & $103.5 \pm 16.96$ & $<0.05$ \\
\hline$R_{\text {tot }} \%$ & $413.40 \pm 329.12$ & $88.30 \pm 29.65$ & $<0.05$ \\
\hline$R_{\text {eff }} \%$ & $348.79 \pm 248.79$ & $72.01 \pm 22.44$ & $<0.05$ \\
\hline
\end{tabular}

Table 1: Summary of clinical characteristics of patients and control individuals. Data are displayed an mean $\pm \mathrm{SD}$, t-test was used for testing for significant differences.

limitation was that wash-out measurements were not possible in patients with the need of oxygen.

Only a small number of patients with GOLD IV $(n=6)$ completed the study (50\% of patients with COPD GOLD IV). Except for two subjects most of our young and healthy controls were fit to complete the measurements without any disturbances $(n=30)$.

\section{The LCI correlated with airway obstruction but failed to discriminate between GOLD I-IV}

The LCI of patients with COPD was significantly higher as compared to healthy and young controls $(\mathrm{p}<0.05)$ (Figure 1). Correlation analysis between spirometric airflow obstruction and the LCI showed negative association between the LCI and the $\mathrm{FEV}_{1}$ (\% pred.) $\left(\mathrm{r}^{2}=0.540, \mathrm{p}<0.01\right)$ (Figure 2a), MEF75 $\left(\mathrm{r}^{2}=0.458, \mathrm{p}<0.01\right)$ (Figure $\left.2 \mathrm{~b}\right), \operatorname{MEF50}\left(\mathrm{r}^{2}=0.403\right.$, $\mathrm{p}<0.01)$ (Figure 2c), MEF25 $\left(\mathrm{r}^{2}=0.309, \mathrm{p}<0.01\right)$ (Figure $2 \mathrm{~d}$ ), and a positive correlation between the $\mathrm{LCI}$ and $\mathrm{R}_{\mathrm{tot}}\left(\%\right.$ pred.) $\left(\mathrm{r}^{2}=0.504\right.$, $\mathrm{p}<0.01$ ) in the whole study population (young and healthy plus elderly patients with COPD) (Figure 2e). Correlation analysis limited to
A

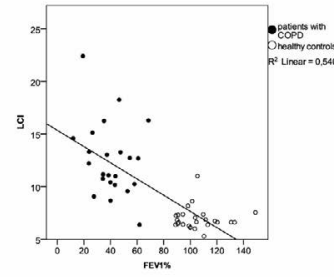

c

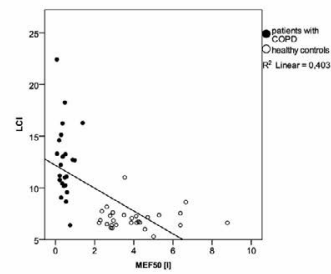

E

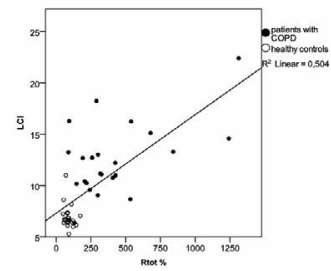

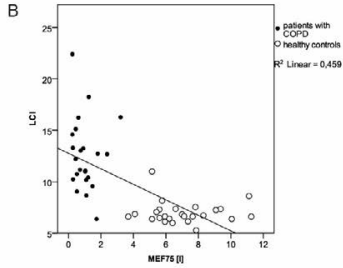

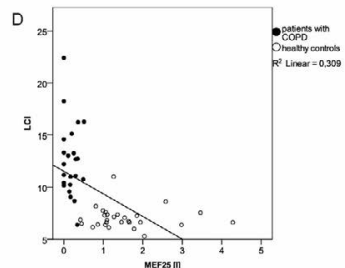

Figure 2: The $\mathrm{LCl}$ correlates with pulmonary function measurements of airflow limitation including $\mathrm{FEV}_{1} \%$ pred. $(A)(p<0.01)$, MEF75 $(B)(p<0.01)$ MEF50 (C) $(p<0.01), \operatorname{MEF} 25$ (D) $(p<0.01)$, and the $R_{\text {tot }} \%$ (total resistance, percent of predicted). $R^{2}$ is indicated in the graph

COPD patients showed a significant correlation between the LCI and $\mathrm{R}_{\text {tot }}\left(\%\right.$ pred.) $\left(\mathrm{r}^{2}=0.295, \mathrm{p}<0.05\right)$ (not shown). There was no significant correlation between the LCI and $\mathrm{FEV}_{1}$ (\% pred.), MEF 75\%, MEF 50\%, and MEF 25\% in the COPD group. These data show that LCI was significantly increased in patients with COPD compared to young and 
healthy controls and this was correlated with spirometric measures of airway obstruction and airway resistance. Overall group comparison of median LCI between healthy controls and the three groups GOLD II-IV was significant $\mathrm{p}<10^{-3}$. Within the elderly patients with COPD there were no significant differences of median LCI between GOLD II, III and IV (pairwise comparisons between GOLD II and III as well as GOLD III and IV). GOLD I was not considered because of the small sample size $n=1$ (no sample representation for the population).

\section{The LCI correlated with hyperinflation but failed to show significant differences between elderly patients with COPD GOLD I-IV}

Pulmonary hyperinflation and ventilation inhomogeneity is a hallmark of COPD and is correlated to clinical outcomes [8]. We asked whether the LCI is correlated with measurements of hyperinflation obtained from body plethysmography. FRC (\% pred.), ITGV (\% pred.) and RV (\% pred.) were significantly elevated in patients with COPD as compared to normal controls $(\mathrm{p}<0.01)$. We found that LCI was correlated with the volume of trapped gas in patients with COPD. LCI showed significant correlation with FRC (\% pred.) $\left(\mathrm{r}^{2}=0.191, \mathrm{p}<0.01\right)$ (Figure 3a), ITGV (\% pred.) $\left(\mathrm{r}^{2}=0.477, \mathrm{p}<0.01\right)$ (Figure $\left.3 \mathrm{~b}\right)$ and RV $(\%$ pred.) $\left(\mathrm{r}^{2}=0.462, \mathrm{p}<0.01\right)$ (Figure $\left.3 \mathrm{c}\right)$ in the whole study population. Correlation analysis limited to elderly COPD patients showed no significant correlation between the LCI and FRC (\% pred.), ITGV ( $\%$ pred.), and RV (\% pred.) (data not shown).

\section{The LCI was not correlated to patient related outcomes}

We next asked whether the LCI was correlated with other outcomes such as the Charlson comorbiditiy index, the number of exacerbations, the SGRQ scores, and the walking distance in the 6-MWT. The LCI neither showed a significant correlation to the Charlson comorbiditiy index $\left(n=23, r^{2}=0.020, p=n . s.\right)$ nor to the number of exacerbations $\left(\mathrm{n}=15, \mathrm{r}^{2}=0.00, \mathrm{p}=\mathrm{n} . \mathrm{s}\right.$.). LCI measurements showed a trend to increase with higher SGRQ ( $\left.\mathrm{n}=8, \mathrm{r}^{2}=0.402, \mathrm{p}=0.092\right)$. No significant relationship between the LCI and the walking distance of the 6-MWT could be detected $\left(n=16, r^{2}=0.027, p=n\right.$. s. $)$.

\section{Discussion}

The main findings of the present study are that LCI measurements are feasible in patients with COPD and that the LCI is increased as compared to normal individuals. LCI measurements were associated with airway obstruction $\left(\mathrm{FEV}_{1}, \mathrm{MEF} 75, \mathrm{MEF} 50, \mathrm{MEF} 25\right.$, and $\left.\mathrm{R}_{\text {tot }}\right)$ and with pulmonary hyperinflation (FRC\%, ITGV\%). Even though LCI measurement is possible in patients with COPD and capable of detecting inhomogeneous ventilation (airway obstruction and hyperinflation), the method has some disadvantages: Compared to body plethysmography, the LCI measurements are more time consuming: Each measurement took more than 20 minutes (dependent on the grade of ventilation inhomogeneity). Moreover, this method excludes patients with severe respiratory failure: Measurements of patients with concurrent oxygen application resulted in invalid measurements. Especially patients with high grade impairment of the lung function refused LCI measurements because the inhalation of dry oxygen gas seemed to be uncomfortable. Moreover, measurements were interrupted by cough several times which lead-as well as leakages at the mouth piece-to invalid measurements. This explained the high drop-out rate of COPD patients (18 out of 41) in our study. Only a small number of patients with GOLD IV completed the study which led to a too small number of patients to draw definite conclusions. This could explain why we could not find any significant correlations between LCI and the different FEV , stages of COPD (LCI was stable and did not increase with decreasing $\mathrm{FEV}_{1}$ ). In addition, there
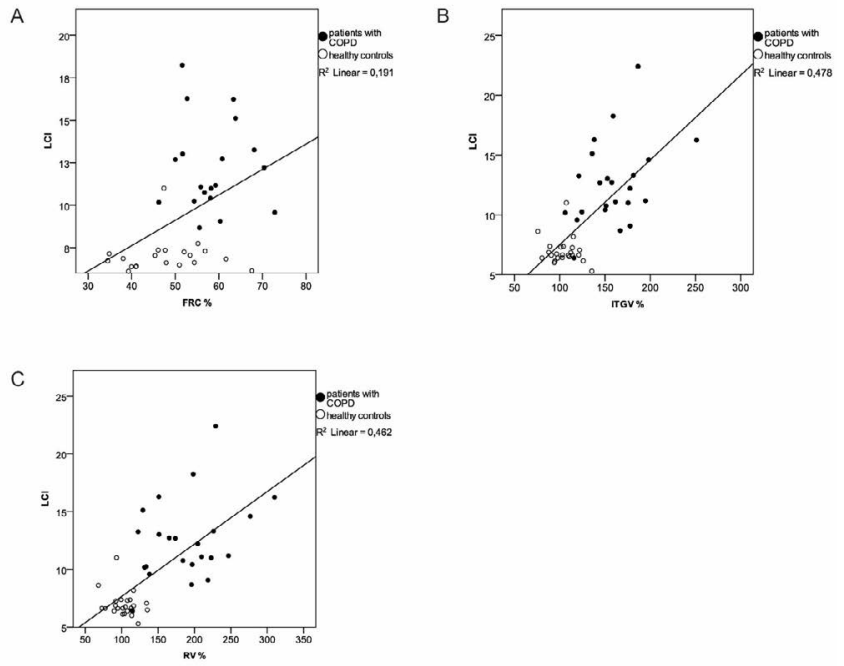

Figure 3: The LCl correlates with hyperinflation represented by the bodyplethysmographic measurements FRC\% pred. (A) $(p<0.01)$, ITGV \% pred. (B) $(p<0.01)$, and $R \vee \%$ pred. $(C)(p<0.01)$.

was no significant correlation between LCI and 6-MWT, an important clinical outcome parameter. Currently the data on LCI measurements in adults and patients with COPD are limited. As one of the advantages of LCI measurement is its non-invasiveness, the recent studies focus on children with cystic fibrosis or asthma [11-16]. The results of the present investigation on healthy adults $(7.58 \pm 1.57)$ are in concordance with data from a $\mathrm{N}_{2}$ based LCI study in 284 healthy subjects [21], which showed a mean LCI for the age groups: $7-19$ yrs: $6.54 \pm 0.28$; $20-39$ yrs; $6.70 \pm 0.36 ; 40-59$ yrs: $7.28 \pm 0.43 ; 60-70$ yrs: $7.78 \pm 0.62$. Other studies described the LCI in healthy adults with $6.94 \pm 0.64(\mathrm{n}=40$, age $40.7 \pm$ $10.3 \mathrm{yrs})$ [17] and $6.7 \pm 0.4(\mathrm{n}=48$, age $33(19-58))$ [13]. According to other studies, we could detect a positive correlation between the LCI and the age as described earlier [22].

In conclusion, the present study gives information about the feasibility of LCI measurement in patients with COPD in daily routine. The LCI is increased in all FEV stages investigated (II-IV) and correlates with airway obstruction and hyperinflation. However, lung clearance measurements require a large effort by the elderly COPD patients, which may be difficult for application in daily routine. Nevertheless, LCI might be a tool to gain insight into lung pathophysiology in patients with COPD. Our preliminary study encourages further investigations for the future.

\section{Acknowledgment}

The abstract of this paper was presented at the ERS International Congress, 26-30 September 2015 in Amsterdam, Netherlands as a poster presentation with interim findings. The poster's abstract was published in "Poster Abstracts" in European Respiratory Journal: http://erj.ersjournals.com/content/46/suppl_59/ PA3354 .

\section{References}

1. Barnes PJ (2000) Chronic obstructive pulmonary disease. N Engl J Med 343 269-280.

2. Cazzola M, MacNee W, Martinez FJ, Rabe KF, Franciosi LG, et al. (2008) Outcomes for COPD pharmacological trials: from lung function to biomarkers. Eur Respir J 31: 416-469.

3. Anthonisen NR, Lindgren PG, Tashkin DP, Kanner RE, Scanlon PD, et al (2005) Bronchodilator response in the lung health study over $11 \mathrm{yrs}$. Eur Respir J 26: 45-51. 
Citation: Fähndrich S, Lepper PM, Trudzinski F, Seibert M, Wagenpfeil S, et al. (2016) Lung Clearance Index is Increased in Patients with COPD - LCI Measurements in the Daily Routine. J Pulm Respir Med 6: 354. doi:10.4172/2161-105X.1000354

4. Jones P, Miravitlles M, van der Molen T, Kulich K (2012) Beyond FEV(1) in COPD: a review of patient-reported outcomes and their measurement. Int $\mathrm{J}$ Chron Obstruct Pulmon Dis 7: 697-709.

5. Bernspång $\mathrm{E}$, Diaz $\mathrm{S}$, Stoel $\mathrm{B}$, Wollmer $\mathrm{P}$, Sveger $\mathrm{T}$, et al. (2011) CT lung densitometry in young adults with alpha-1-antitrypsin deficiency. Respir Med 105: 74-79.

6. Galban CJ, Han MK, Boes JL, Chughtai KA, Meyer CR, et al. (2012) Computed tomography-based biomarker provides unique signature for diagnosis of COPD phenotypes and disease progression. Nat Med 18: 1711-1715.

7. Celli BR, Locantore N, Yates J, Tal-Singer R, Miller BE, et al. (2012) Inflammatory biomarkers improve clinical prediction of mortality in chronic obstructive pulmonary disease. Am J Respir Crit Care Med 185: 1065-1072.

8. Robinson PD, Latzin P, Verbanck S, Hall GL, Horsley A, et al. (2013) Consensus statement for inert gas washout measurement using multiple- and singlebreath tests. Eur Respir J 41: 507-522.

9. Becklake MR (1952) A new index of the intrapulmonary mixture of inspired air. Thorax 7: 111-116.

10. Horsley A (2009) Lung clearance index in the assessment of airways disease Respir Med 103: 793-799.

11. Stahl M, Joachim C, Blessing K, Hämmerling S, Sommerburg O, et al. (2014) Multiple breath washout is feasible in the clinical setting and detects abnormal lung function in infants and young children with cystic fibrosis. Respiration 87 357-363.

12. Horsley AR, Macleod KA, Robson AG, Lenney J, Bell NJ, et al. (2008) Effects of cystic fibrosis lung disease on gas mixing indices derived from alveolar slope analysis. Respir Physiol Neurobiol 162: 197-203.

13. Horsley AR, Gustafsson PM, Macleod KA, Saunders C, Greening AP, et al. (2008) Lung clearance index is a sensitive, repeatable and practical measure of airways disease in adults with cystic fibrosis. Thorax 63: 135-140.

14. Fuchs SI, Buess C, Lum S, Kozlowska W, Stocks J, et al. (2005) Multiple breath washout with a sidestream ultrasonic flow sensor and mass spectrometry: a comparative study. Pediatr Pulmonol 41: 1218-1225.

15. Subbarao P, Stanojevic S, Brown M, Jensen R, Rosenfeld M, et al. (2013) Lung clearance index as an outcome measure for clinical trials in young children with cystic fibrosis. A pilot study using inhaled hypertonic saline. Am J Respir Crit Care Med 188: 456-460.

16. Keen C, Olin AC, Wennergren G, Gustafsson P (2011) Small airway function exhaled NO and airway hyper-responsiveness in paediatric asthma. Respir Med 105: 1476-1484.

17. Husemann K, Berg N, Engel J, Port J, Joppek C, et al. (2014) Double tracer gas single-breath washout: reproducibility in healthy subjects and COPD. Eur Respir J 44: 1210-1222.

18. Miller MR, Hankinson J, Brusasco V, Burgos F, Casaburi R, et al. (2005) Standardisation of spirometry. Eur Respir J 26: 319-338.

19. Macintyre N, Crapo RO, Viegi G, Johnson DC, van der Grinten CP, et al. (2005) Standardisation of the single-breath determination of carbon monoxide uptake in the lung. Eur Respir J 26: 720-735.

20. Sharabiani MT, Aylin P, Bottle A (2012) Systematic review of comorbidity indices for administrative data. Med Care 50: 1109-1118.

21. Houltz B, Green K, Lindblad A, Singer F, Nielsen K, et al. (2012) Titad N washout ventilation inhomogeineity indices in a reference population ages 7-70years. Eur Respir J 40: 694s.

22. Verbanck S, Thompson BR, Schuermans D, Kalsi H, Biddiscombe M, et al (2012) Ventilation heterogeneity in the acinar and conductive zones of the normal ageing lung. Thorax 67: 789-795. 